\title{
Ectopic bronchogenic cyst in the retroperitoneal region: a case report and literature review of adult patients
}

\author{
Kaitao Yuan ${ }^{1+}$, Man Shu ${ }^{2+}$, Yan Ma ${ }^{1}$, Weidong Feng ${ }^{1}$, Jinning Ye ${ }^{1}$ and Yujie Yuan ${ }^{1 *}$ (D)
}

\begin{abstract}
Background: Bronchogenic cyst is congenital aberration of bronchopulmonary malformation, which is rarely encountered in the abdomen and retroperitoneum. We present a case report and literature review of retroperitoneal bronchogenic cyst.

Case presentation: A 53-year-old female presented to outpatient clinic for a routine checkup of lumbar intervertebral disc herniation. She received a contrast computed tomography scan of the abdomen which revealed a retroperitoneal cystic lesion below the left crura of diaphragm. Afterward, the patient underwent a laparoscopic excision of the cystic lesion and was discharged uneventfully at postoperative day 4 . Histopathological findings confirmed the diagnosis of retroperitoneal bronchogenic cyst. Our literature review identified 55 adult cases in recent two decades. The average age at diagnosis was 43.2 (range 17-69) years. 44 (80\%) cases had a retroperitoneal cyst on the left side, and 52 (94.5\%) cases underwent curative excision through open or laparoscopic surgery. In the available follow up of cases, there was no recurrence after surgery.
\end{abstract}

Conclusions: Bronchogenic cyst is rare in the retroperitoneal region. It should be considered as one of the differential diagnoses of a retroperitoneal neoplasm.

Keywords: Retroperitoneal neoplasm, Bronchogenic cyst, Case report, Literature review, Surgical management

\section{Background}

Bronchogenic cyst is rare, benign congenital malformation of the tracheobronchial tree within the early embryologic foregut period [1]. It is mostly discovered in the posterior mediastinum but rarely found in the retroperitoneal region. Retroperitoneal bronchogenic cyst is first reported by Miller et al. in 1953 [2]. Since such cyst is usually asymptomatic, its diagnosis before surgery in such location is still challenging. We here present

\footnotetext{
*Correspondence: yuanyj5@mail.syu.edu.cn

${ }^{\dagger}$ Kaitao Yuan and Man Shu have equally contributed to this study

${ }^{1}$ Center of Gastrointestinal Surgery, The First Affiliated Hospital, Sun

Yat-sen University, No. 58 2nd Zhongshan Road, Guangzhou 510080,

Guangdong Province, People's Republic of China

Full list of author information is available at the end of the article
}

a woman with incidental detection of a retroperitoneal bronchogenic cyst, which was successfully managed through a laparoscopic excision. Additionally, we have performed a literature review to update the clinical features of this rare disease in adult patients.

\section{Case presentation}

A 53-year-old female patient presented to the outpatient clinic of local hospital in June 2017 (day 0) for routine checkup of lumbar intervertebral disc herniation. She underwent a magnetic resonance imaging (MRI) scan that revealed a round small mass in the retroperitoneal space. On day 3, she was referral to our department for further diagnosis and treatment of the retroperitoneal neoplasm. She did not have any complaint except for disc herniation-related low back pain. Her past history and 
family history were non-contributory. Physical examination was insignificant. Routine laboratory studies were normal. Specifically, serum tumor markers including carbohydrate antigen 19-9 (CA19-9), carbohydrate antigen 125 (CA125) and carcinoembryonic antigen (CEA) were all within normal ranges. Afterward, she received abdominal contrast-enhanced computed tomography $(\mathrm{CT})$ scans, which revealed a well circumscribed cystic lesion, measuring $3.3 \times 2.7 \times 3.5 \mathrm{~cm}^{3}$ and filling with non-enhancing fluid-density collections, in her retroperitoneal region (Fig. 1). The lesion was located below the diaphragm and was adjacent to the left crura of diaphragm and abdominal aorta. After a multidisciplinary team discussion, a minimally invasive surgery was planned for her to determine the feature of such cystic lesion. On day 12, the patient underwent laparoscopic exploration and lesion excision. The cystic lesion, smoothly surfaced with mucinous content, was totally separated from the left crura of diaphragm, abdominal aorta, stomach, and left adrenal gland. The operation was successfully performed, which lasted for $90 \mathrm{~min}$ with estimated blood loss $10 \mathrm{~mL}$. Oral feeding started $6 \mathrm{~h}$ after surgery, and the postoperative recovery was uneventful. On day 16, the patient was discharged uneventfully from the hospital. She did not develop any complications at 3-month follow-up. Her histopathological findings indicated that the cystic wall consisted of ciliated pseudostratified epithelium, smooth muscle, seromucous glands and fully developed cartilage (Fig. 2). Thus, the patient had a confirmed diagnosis of retroperitoneal bronchogenic cyst. She continued her daily activities without any limitation and had no evidence of recurrence within two years of follow-up.

\section{Discussion and conclusions}

Bronchogenic cyst was defined with the following criteria: pseudo-stratified, ciliated, columnar epithelium together with the existence of at least one of the following structures: cartilage, smooth muscle or seromucous glands [3]. It commonly occurred in the mediastinal region of the thorax, while rarely located in the retroperitoneal region. To review this uncommon disease, we
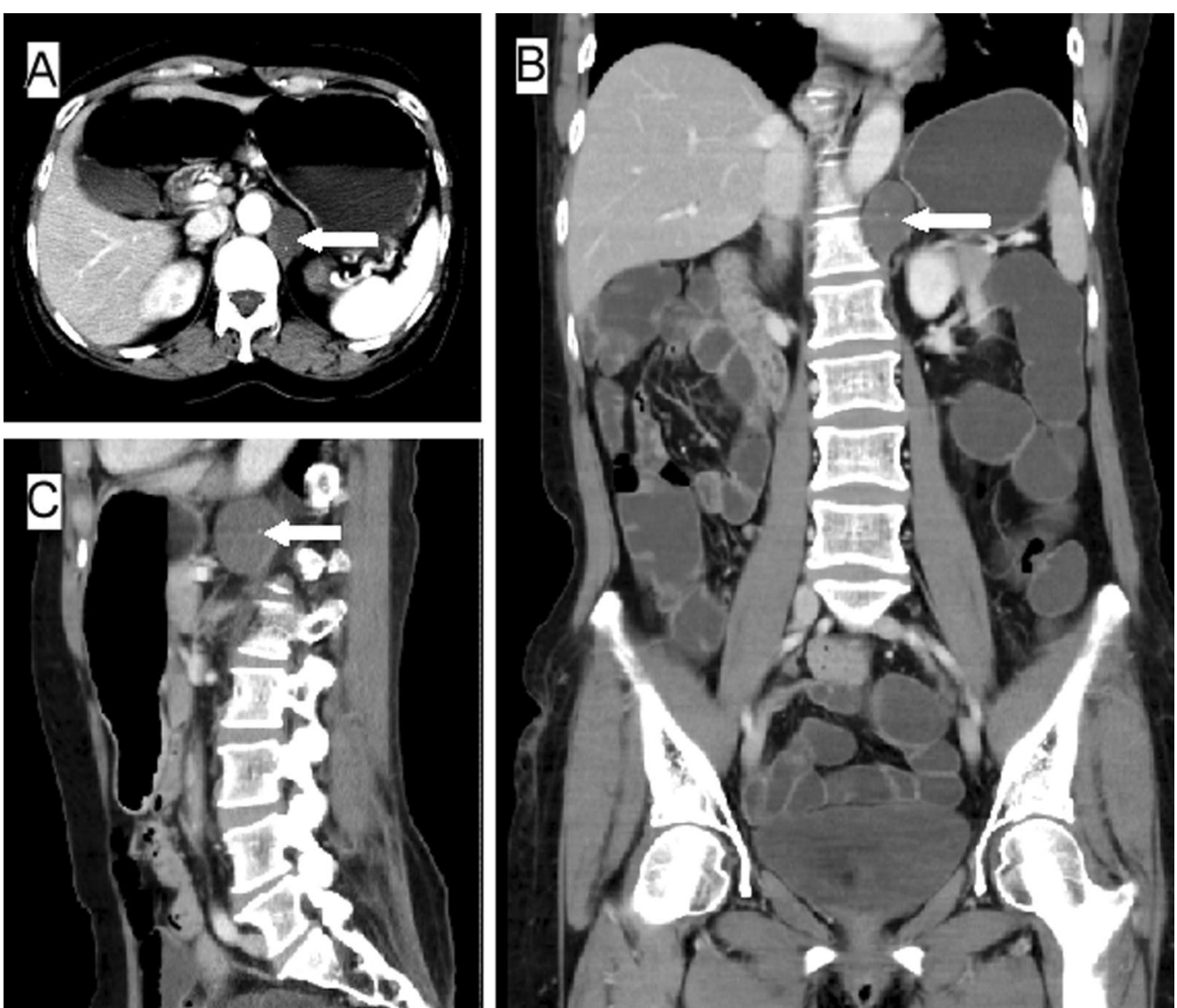

Fig. 1 Radiological findings of the reported case. Contrast-enhanced CT scan shows a thin-walled water-attenuated cystic lesion in retroperitoneal region (white arrows). Panel A, Axial view; Panel B, Coronal view; Panel C, Sagittal view 


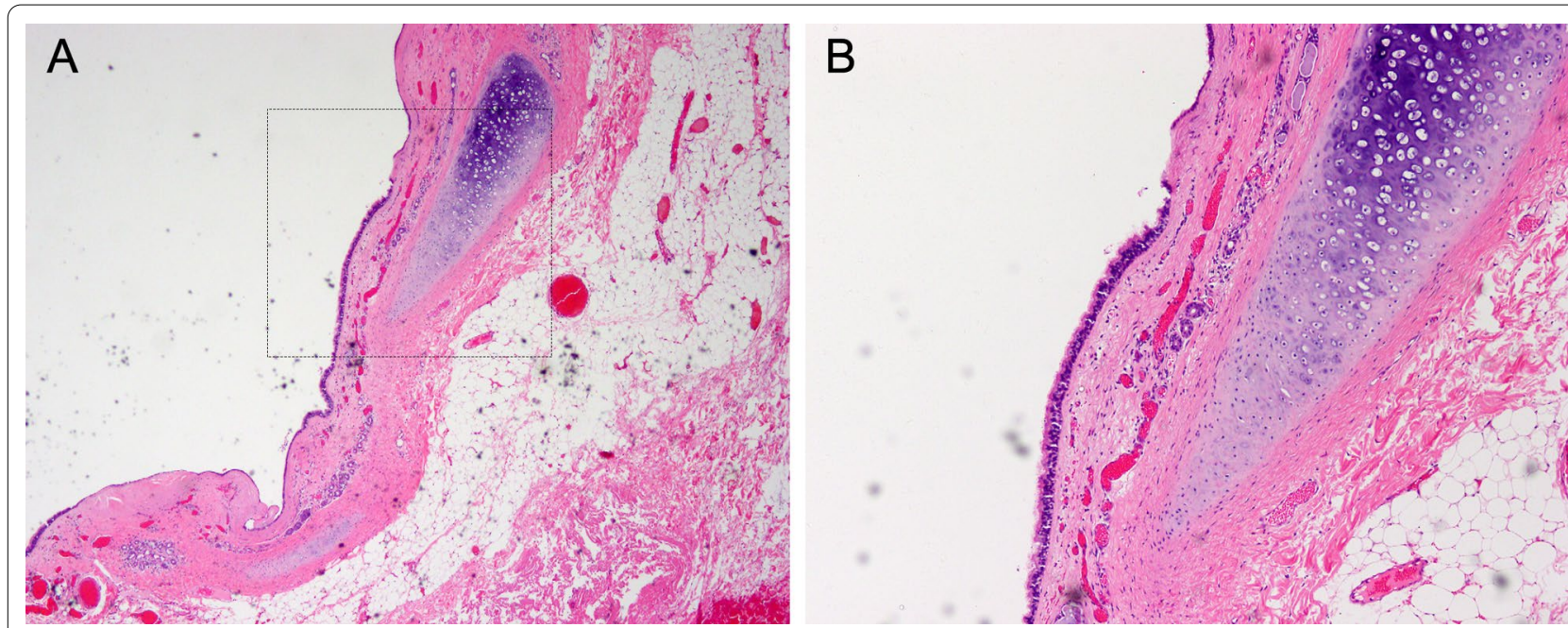

Fig. 2 Microscopic appearance of retroperitoneal bronchogenic cyst. The cystic wall was consisted of pseudostratified epithelium, smooth muscle, mixed seromucous glands and cartilage. (Hematoxylin and eosin stain, original magnification: $\mathbf{A} \times 40$ and $\mathbf{B} \times 200$ power fields; The dotted box indicates magnified field)

searched PubMed and Embase databases for similar case reports published between January 1998 and January 2018. The used keywords were as follows: "retroperitoneal", "bronchogenic", "mass", "tumor", "neoplasm" and "cyst". English language studies with adult patient populations ( $\geq 18$ years) were reviewed by two surgeons (WF and JY). The clinical data of selected cases with special attention to primary symptoms, tumor size and location, and histopathological features were summarized.

In sum, 55 cases ( 24 female and 31 male) with retroperitoneal bronchogenic cyst were identified within the study period. The clinical characteristics of included cases are shown in Table 1 . The average age at diagnosis of retroperitoneal bronchogenic cyst was 43.2 (range 17-69) years old. The primary complaints included asymptomatic (27 cases), abdominal discomfort (12 cases), left flank pain (7 cases) and back pain (5 cases). The average diameter of retroperitoneal bronchogenic cyst was 6.4 (range 2-20) $\mathrm{cm}$. Most of those cysts (44 cases) were found in the left retroperitoneal region, with eight cases discovered on the right side. The serum level of CA19-9 was elevated in only two cases $[4,5]$. There was one case reported CEA level elevated inside the cyst but normal in the serum [6]. Laparoscopic resection was performed in most of the reported patients as the increased popularity of minimally invasive surgery. There were only two cases who received biopsy procedures and routine follow-up visits $[7,8]$. For those obtaining a curative resection, postoperative recurrence was not reported.

A similar literature review was conducted by Govaerts et al. in 2012, which concisely summarized
30 cases of true retroperitoneal bronchogenic cysts [2]. To our knowledge, our review provides the largest case series of adult patients with such cysts. Unfortunately, the exact pathogenesis of retroperitoneal bronchogenic cyst is still undetermined. More than 30 years ago, it was hypothesized that the pleuroperitoneal membrane and embryonic diaphragm might have not yet fused in an early development stage [1]. Consequently, abnormal tracheobronchial buds could be isolated by the growing diaphragm and migrate into abdominal cavity, which finally develop into bronchogenic cysts in the retroperitoneal space. Since the left pericardioperitoneal canal is larger and closes later than the right one, $80 \%$ of reported cases located in the left side of the retroperitoneal region, as in our reported case. According to the included cases (Table 1), the most common location of retroperitoneal bronchogenic cyst is near the left adrenal gland, and the second most common location is the peripancreatic region. There is a case of bilateral adrenal multilocular retroperitoneal bronchogenic cysts [9]. Retroperitoneal bronchogenic cyst occurs with equal frequency in men and women, with an average diagnosis age of 43.2 (range 17-69) years.

As mentioned above, the majority of reported retroperitoneal bronchogenic cysts were asymptomatic and discovered incidentally as our case. However, some specific cysts were discovered when causing certain symptoms, such as infection, uncomfortable abdomen and vomiting, from compression to adjacent organs. Patients usually complain epigastric pain or left flank pain when the cyst is over $5 \mathrm{~cm}$ in diameter. Up to date, Mirsadeghi et al. reported the largest retroperitoneal 
Table 1 Literature review of retroperitoneal bronchogenic cyst

\begin{tabular}{|c|c|c|c|c|c|c|c|c|c|}
\hline No. & Study & Country & Gender & Age & $\begin{array}{l}\text { Primary } \\
\text { symptoms }\end{array}$ & $\begin{array}{l}\text { Maximal } \\
\text { size }(\mathrm{cm})\end{array}$ & Location & Treatment & Specific features \\
\hline 1 & Buckley 1998 [16] & USA & $\mathrm{F}$ & 46 & $\begin{array}{l}\text { Epigastric pain and } \\
\text { weight loss }\end{array}$ & 4 & Left adrenal gland & Laparotomy & \\
\hline 2 & $\begin{array}{l}\text { Yamamoto } 1998 \\
\text { [17] }\end{array}$ & Japan & $\mathrm{F}$ & 49 & No & 3 & Right adrenal glad & Laparoscopic & \\
\hline 3 & Itoh 1999 [18] & Japan & $\mathrm{F}$ & 46 & $\begin{array}{l}\text { Pain in the left } \\
\text { flank }\end{array}$ & 8 & Left adrenal glad & Laparotomy & \\
\hline 4 & Sullivan 1999 [19] & Japan & $\mathrm{F}$ & 55 & $\begin{array}{l}\text { Lower abdominal } \\
\text { discomfort }\end{array}$ & 10 & $\begin{array}{l}\text { Retroperitoneal to } \\
\text { ascending colon }\end{array}$ & Laparotomy & \\
\hline 5 & Yang 1999 [20] & South Korea & M & 30 & No & 6 & Left adrenal glad & Laparotomy & \\
\hline 6 & Reichelt 2000 [21] & Germany & M & 46 & No & 3.8 & $\begin{array}{l}\text { Right retroperito- } \\
\text { neum }\end{array}$ & Laparotomy & \\
\hline 7 & $\begin{array}{l}\text { Haddadin } 2001 \\
{[22]}\end{array}$ & UK & M & 51 & Epigastric pain & 4 & $\begin{array}{l}\text { Left suprarenal } \\
\text { region }\end{array}$ & Laparotomy & \\
\hline 8 & $\begin{array}{l}\text { Anderson } 2001 \\
{[23]}\end{array}$ & USA & M & 33 & $\begin{array}{l}\text { Left flank pain and } \\
\text { gross hematuria }\end{array}$ & 6 & $\begin{array}{l}\text { Left suprarenal } \\
\text { region }\end{array}$ & Laparotomy & $\begin{array}{l}\text { Adenocarcinoma } \\
\text { with P53 positive }\end{array}$ \\
\hline 9 & Martin 2002 [24] & Spain & M & 51 & No & 8 & $\begin{array}{l}\text { Left diaphragmic } \\
\text { pillar }\end{array}$ & Laparotomy & \\
\hline 10 & Ingu 2002 [25] & Japan & $\mathrm{F}$ & 46 & $\begin{array}{l}\text { Progressive left- } \\
\text { arm numbness }\end{array}$ & 4 & $\begin{array}{l}\text { Left hemidi- } \\
\text { aphragm }\end{array}$ & Laparotomy & \\
\hline 11 & $\begin{array}{l}\text { Andersson } 2003 \\
\text { [26] }\end{array}$ & Sweden & M & 38 & $\begin{array}{l}\text { Upper abdominal } \\
\text { pain and weight } \\
\text { loss }\end{array}$ & 4.5 & $\begin{array}{l}\text { Left posteriors to } \\
\text { pancreas }\end{array}$ & Laparotomy & $\begin{array}{l}\text { No recurrence } \\
2 \text { years follow-up }\end{array}$ \\
\hline 12 & Ishikawa 2003 [15] & Japan & $\mathrm{F}$ & 41 & Left flank pain & 9.2 & Left adrenal gland & $\begin{array}{l}\text { Retroperitoneo- } \\
\text { scopic }\end{array}$ & \\
\hline 13 & Hedayati 2003 [27] & USA & $\mathrm{F}$ & 59 & No & 7 & Left adrenal gland & Laparotomy & \\
\hline 14 & Hisatomi 2003 [28] & Japan & M & 42 & Left flank pain & 12 & $\begin{array}{l}\text { Left retroperito- } \\
\text { neal space }\end{array}$ & Laparotomy & \\
\hline 15 & Ishizuka 2004 [29] & Japan & M & 36 & No & 5 & Left adrenal glad & Laparoscopic & \\
\hline 16 & Goh 2004 [30] & Singapore & F & 29 & $\begin{array}{l}\text { A right-sided } \\
\text { abdominal mass }\end{array}$ & 18.9 & $\begin{array}{l}\text { Right retroperito- } \\
\text { neal region }\end{array}$ & Laparotomy & \\
\hline 17 & Paik 2005 [31] & South Korea & M & 59 & No & 7 & $\begin{array}{l}\text { Superior border of } \\
\text { pancreas neck }\end{array}$ & Laparotomy & Colon cancer \\
\hline 18 & Wang 2006 [32] & Taiwan & M & 69 & $\begin{array}{l}\text { Right upper quad- } \\
\text { rant discomfort }\end{array}$ & 7 & $\begin{array}{l}\text { Left anterior para- } \\
\text { renal space }\end{array}$ & Laparotomy & \\
\hline 19 & Kim 2007 [33] & South Korea & M & 17 & Abdominal pain & 3.2 & $\begin{array}{l}\text { Left anterior para- } \\
\text { renal space }\end{array}$ & Laparotomy & \\
\hline 20 & Chu 2007 [34] & Taiwan & M & 55 & No & 4 & Left adrenal gland & Laparoscopic & \\
\hline 21 & Roma 2008 [35] & USA & M & 40 & No & 6.2 & Left adrenal gland & Laparoscopic & \\
\hline 22 & Chung 2009 [36] & South Korea & F & 41 & No & 4.8 & Left adrenal gland & $\begin{array}{l}\text { Retroperitoneo- } \\
\text { scopic }\end{array}$ & \\
\hline 23 & Obando 2009 [37] & USA & M & 67 & No & 3.9 & $\begin{array}{l}\text { Between pancreas } \\
\text { and stomach }\end{array}$ & Laparoscopic & \\
\hline 24 & Önol 2009 [38] & Turkey & M & 36 & No & 6 & Left adrenal & Laparotomy & \\
\hline 25 & Inaba 2010 [39] & Japan & $\mathrm{F}$ & 64 & No & 4 & $\begin{array}{l}\text { Posterior wall of } \\
\text { stomach }\end{array}$ & Laparoscopic & \\
\hline 26 & $\begin{array}{l}\text { El Youssef } 2010 \\
\text { [40] }\end{array}$ & Portland & M & 44 & No & 3 & Left adrenal mass & Laparoscopic & \\
\hline 27 & Diaz 2010 [41] & Spain & M & 67 & Low back pain & 6 & $\begin{array}{l}\text { GEJ to left dia- } \\
\text { phragmatic crura }\end{array}$ & Laparoscopic & \\
\hline 28 & Petrina 2010 [42] & Italy & M & 33 & $\begin{array}{l}\text { Epigastric abdomi- } \\
\text { nal pain }\end{array}$ & 5 & Ileal mesentery & Laparotomy & \\
\hline 29 & $\begin{array}{l}\text { Alguraan } 2012 \\
\text { [43] }\end{array}$ & USA & $\mathrm{F}$ & 23 & No & 4 & $\begin{array}{l}\text { Right adrenal } \\
\text { mass }\end{array}$ & $\begin{array}{l}\text { Retroperitoneo- } \\
\text { scopic }\end{array}$ & Robotic excision \\
\hline 30 & Parray 2012 [44] & India & $\mathrm{F}$ & 30 & $\begin{array}{l}\text { Right upper quad- } \\
\text { rant pain }\end{array}$ & 10 & $\begin{array}{l}\text { Right suprarenal } \\
\text { area }\end{array}$ & Laparotomy & \\
\hline
\end{tabular}


Table 1 (continued)

\begin{tabular}{|c|c|c|c|c|c|c|c|c|c|}
\hline No. & Study & Country & Gender & Age & $\begin{array}{l}\text { Primary } \\
\text { symptoms }\end{array}$ & $\begin{array}{l}\text { Maximal } \\
\text { size }(\mathrm{cm})\end{array}$ & Location & Treatment & Specific features \\
\hline 31 & O’Neal 2012 [45] & USA & $\mathrm{F}$ & 23 & $\begin{array}{l}\text { Vague abdominal } \\
\text { discomfort }\end{array}$ & 5.2 & Left adrenal gland & $\begin{array}{l}\text { Retroperitoneo- } \\
\text { scopic }\end{array}$ & \\
\hline 32 & Brient 2012 [7] & France & $M$ & 60 & No & NA & $\begin{array}{l}\text { Left diaphragmic } \\
\text { pillar }\end{array}$ & CT-guided biopsy & $\begin{array}{l}\text { No recurrence } \\
3 \text { years follow-up }\end{array}$ \\
\hline 33 & Govaerts 2012 [2] & Belgium & M & 48 & No & 7.5 & Left adrenal glad & Laparotomy & \\
\hline 34 & Choi 2012 [14] & South Korea & $\mathrm{F}$ & 45 & $\begin{array}{l}\text { Intermittent } \\
\text { abdominal dis- } \\
\text { comfort }\end{array}$ & 8 & Left adrenal gland & Laparotomy & \\
\hline 35 & Runge 2013 [6] & Switzerland & $F$ & 42 & Epigastric pain & 5.0 & Left adrenal glad & Laparoscopic & $\begin{array}{l}\text { Inside CEA } \\
3777 \mu \mathrm{g} / \mathrm{L}\end{array}$ \\
\hline 36 & Cai 2013 [46] & China & $\mathrm{F}$ & 26 & No & 4 & Left adrenal region & Laparotomy & \\
\hline 37 & Castro 2013 [47] & Portugal & $\mathrm{F}$ & 36 & Abdominal pain & 8 & $\begin{array}{l}\text { Left upper retrop- } \\
\text { eritoneum }\end{array}$ & Laparoscopic & \\
\hline 38 & Jannasch 2013 [4] & Germany & M & 50 & $\begin{array}{l}\text { In the left flank } \\
\text { pain }\end{array}$ & 4 & Left adrenal region & $\begin{array}{l}\text { Retroperitoneo- } \\
\text { scopic }\end{array}$ & CA19-9 144.1 \\
\hline 39 & Kluger 2013 [48] & USA & $\mathrm{F}$ & 49 & $\begin{array}{l}\text { Intermittent } \\
\text { epigastric pain }\end{array}$ & 8.5 & $\begin{array}{l}\text { Right side of } \\
\text { pancreas head }\end{array}$ & Laparotomy & $\begin{array}{l}\text { Whipple's proce- } \\
\text { dure }\end{array}$ \\
\hline 40 & Cao 2014 [9] & China & M & 51 & $\begin{array}{l}\text { Intermittent } \\
\text { fatigue }\end{array}$ & 2.1 & Bilateral adrenal & $\begin{array}{l}\text { Retroperitoneo- } \\
\text { scopic }\end{array}$ & \\
\hline 41 & Dong 2014 [49] & China & $\mathrm{F}$ & 30 & No & 2 & $\begin{array}{l}\text { Left suprarenal } \\
\text { region }\end{array}$ & $\begin{array}{l}\text { Retroperitoneo- } \\
\text { scopic }\end{array}$ & CEA elevated inside \\
\hline 42 & $\begin{array}{l}\text { Mirsadeghi } 2014 \\
{[10]}\end{array}$ & Iran & $M$ & 23 & $\begin{array}{l}\text { Left upper quad- } \\
\text { rant pain }\end{array}$ & 20 & $\begin{array}{l}\text { Between spleen } \\
\text { and left kidney }\end{array}$ & Laparotomy & $\begin{array}{l}\text { No recurrence } \\
4 \text { years follow-up }\end{array}$ \\
\hline 43 & Terasaka 2014 [50] & Japan & M & 27 & No & 5.4 & Left adrenal gland & Laparoscopic & \\
\hline 44 & Bulut 2015 [51] & Turkey & $\mathrm{F}$ & 25 & $\begin{array}{l}\text { Pain in the left } \\
\text { flank }\end{array}$ & 4 & Left adrenal gland & Laparoscopic & \\
\hline 45 & Herek 2015 [52] & Turkey & $M$ & 42 & Back pain & 9 & $\begin{array}{l}\text { Left diaphragmic } \\
\text { crura }\end{array}$ & Laparotomy & \\
\hline 46 & Jiang 2015 [53] & China & $M$ & 52 & No & 2.5 & $\begin{array}{l}\text { Left retroperito- } \\
\text { neal space }\end{array}$ & $\begin{array}{l}\text { Retroperitoneo- } \\
\text { scopic }\end{array}$ & \\
\hline 47 & $\begin{array}{l}\text { Robertson } 2015 \\
\text { [54] }\end{array}$ & UK & $M$ & 56 & $\begin{array}{l}\text { Right hypochon- } \\
\text { drial pain }\end{array}$ & 6.6 & $\begin{array}{l}\text { Right hemidi- } \\
\text { aphragm }\end{array}$ & Laparotomy & Mesh repair \\
\hline 48 & Tong 2015 [55] & China & $\mathrm{F}$ & 36 & Lower back pain & 17 & $\begin{array}{l}\text { Left upper retrop- } \\
\text { eritoneal space }\end{array}$ & Laparotomy & \\
\hline 49 & Trehan 2015 [56] & India & $\mathrm{F}$ & 34 & $\begin{array}{l}\text { Heaviness in right } \\
\text { flank }\end{array}$ & 10 & $\begin{array}{l}\text { Right retroperito- } \\
\text { neal hypochon- } \\
\text { drium }\end{array}$ & Laparoscopic & \\
\hline 50 & Yoon 2015 [57] & South Korea & $M$ & 57 & No & 4.8 & Left adrenal gland & Laparoscopic & \\
\hline 51 & Pasquer 2016 [58] & France & $M$ & 36 & No & 2 & Retro-rectal space & Sacrococcygeal & $\begin{array}{l}\text { No recurrence after } \\
3 \text { years }\end{array}$ \\
\hline 52 & Wang 2017 [13] & China & $\mathrm{F}$ & 48 & No & 8 & Left adrenal gland & Laparoscopic & \\
\hline 53 & Byers 2018 [8] & USA & $M$ & 52 & No & 3.1 & Left adrenal gland & FNA & Colon cancer \\
\hline 54 & Liu 2018 [5] & China & $M$ & 33 & No & 4.5 & Left hepatic hilum & Robotic surgery & CA19-9 312iu/m \\
\hline 55 & Yuan $2021^{*}$ & China & $\mathrm{F}$ & 53 & Back pain & 3.5 & Left adrenal gland & Laparoscopic & Normal follow-up \\
\hline
\end{tabular}

NA not available; GEJ gastroesophageal junction; FNA fine needle aspiration; $M$ male; F female; UK United Kingdom; USA United States of American

*Our case

bronchogenic cyst in a 23-year-old man, with a maximal diameter of $20 \mathrm{~cm}$ [10].

In the current literature review, retroperitoneal bronchogenic cysts were confirmed after surgery or invasive biopsy. It remains quite difficult to make correct diagnosis preoperatively. Such cystic lesions would not arouse specific symptoms, therefore, they are easily misdiagnosed as non-neoplastic tumors, such as adrenal adenoma, adrenal cyst and pancreas cyst, or neoplastic tumors, such as adrenal cortical carcinoma, pheochromocytoma and pancreatic adenocarcinoma. Although CT scans and MRI examinations are helpful 
to locate the retroperitoneal masses, they play a minor role in determining the origin of such lesions. In clinical workup, a cystic mass in the retroperitoneal space could account for a broad diagnosis entity, including benign tumors such as lymphangioma, urothelial cyst, microcystic pancreatic adenoma, and other masses such as hematoma, abscess, duplication cyst, ovarian cyst and pancreatic pseudocyst, malignant tumors such as cystic mesothelioma, teratoma, undifferentiated sarcoma, cystic metastases (especially from ovarian or gastric adenocarcinomas), and malignant mesenchymoma [11].

To the present, histopathology is indispensable to clarify a definitive diagnosis of bronchogenic cyst. The essential pathological criteria are the presence of secretory respiratory lining epithelium along with seromucous glands, smooth muscle cells or hyaline cartilage [12]. Of note, all three important structures mentioned above were found in our reported case (Fig. 2).

The relationship between tumor markers and bronchogenic cysts is still unknown. Wang et al. reported a case of retroperitoneal bronchogenic cyst with serum CA 19-9 level more than $1200 \mathrm{U} / \mathrm{mL}$. After 2 months of cyst excision, the CA 19-9 level returned to normal (cutoff: $35 \mathrm{U} / \mathrm{mL}$ ) [13]. Another case with increased serum CA 19-9 level (144.1 U/mL) was reported by Choi et al. [14]. Besides, the CEA level was elevated at $3777 \mu \mathrm{g} / \mathrm{L}$ (cutoff: $5 \mu \mathrm{g} / \mathrm{L}$ ) inside the cyst, but normal at $4.3 \mu \mathrm{g} / \mathrm{L}$ in the serum [6].

In order to confirm the diagnosis, relieve associated symptoms and prevent any potential malignant transformations, surgical resection is suggested for either symptomatic or asymptomatic retroperitoneal bronchogenic cyst [15]. Complete laparoscopic excision of cystic lesion is safe and feasible in our case. Moreover, it improves postoperative discomfort and shortens hospital stay as compared to traditional open approach. In recent years, robotic surgery, which could facilitate precise dissection of retroperitoneal tumors, was successfully applied to excise retroperitoneal bronchogenic cyst [5]. Generally, a complete resection via laparoscopic surgery often earns a good prognosis for retroperitoneal bronchogenic cysts, with no report of recurrence noted in this review.

In the current study, we reported a case with an ectopic bronchogenic cyst in the left retroperitoneal region. The uncommon ectopic cyst should be well known and considered as a part of differential diagnosis for a retroperitoneal neoplasm. A literature review suggests that retroperitoneal laparoscopic excision is an optimal management to establish both diagnosis and treatment. The long-term outcome of this disease is excellent, with no report of recurrence.

\section{Abbreviations}

CT: Computed tomography; MRI: Magnetic resonance imaging; CEA: Carcinoembryonic antigen; CA19-9: Carbohydrate antigen 19-9; CA125: Carbohydrate antigen 125

\section{Acknowledgements}

None.

\section{Authors' contributions}

KY and YY planned and designed the case report and literature review. KY, MS and WF collected and reviewed the literature on the subject and drafted the paper. YY, MS and JY performed the draft revision and collected clinical and pathological data. YY and YM provided critical revision for final manuscript. MS provided pathological images and JY performed the analysis and interpretation of data. All authors read and approved the final manuscript.

Funding

None.

\section{Availability of data and materials}

The datasets used during the current study are available from the corresponding author on reasonable request.

\section{Declarations}

\section{Ethics approval and consent to participate}

Our work was approved by the Ethics Committee of the First Affiliated Hospital of Sun Yat-sen University, and written informed consent was obtained from the patient.

\section{Consent for publication \\ Written consent for publication of this case and clinical dates was informed and obtained from the patient.}

\section{Competing interests}

All authors have declared that no conflict of any interests.

\section{Author details}

${ }^{1}$ Center of Gastrointestinal Surgery, The First Affiliated Hospital, Sun Yat-sen University, No. 58 2nd Zhongshan Road, Guangzhou 510080, Guangdong Province, People's Republic of China. ${ }^{2}$ Department of Pathology, The First Affiliated Hospital, Sun Yat-sen University, Guangzhou 510080, Guangdong Province, People's Republic of China.

Received: 8 March 2021 Accepted: 6 September 2021

Published online: 20 September 2021

\section{References}

1. Sumiyoshi K, Shimizu S, Enjoji M, Iwashita A, Kawakami K. Bronchogenic cyst in the abdomen. Virchows Arch A. 1985;408(1):93-8.

2. Govaerts K, Van Eyken P, Verswijvel G, Van der Speeten K. A bronchogenic cyst, presenting as a retroperitoneal cystic mass. Rare Tumors. 2012;4(1):e13

3. Cuypers P, De Leyn P, Cappelle L, Verougstraete L, Demedts M, Deneffe G. Bronchogenic cysts: a review of 20 cases. Eur J Cardiothorac Surg. 1996;10(6):393-6.

4. Jannasch O, Buschel P, Wodner C, Seidensticker M, Kuhn R, Lippert H, Mroczkowski P. Retroperitoneoscopic and laparoscopic removal of periadrenally located bronchogenic cysts - a systematic review. Pol Przegl Chir. 2013;85(12):706-13.

5. Liu Q, Gao Y, Zhao Z, Zhao G, Liu R, Lau WY. Robotic resection of benign nonadrenal retroperitoneal tumors: a consecutive case series. Int J Surg. 2018;55:188-92.

6. Runge T, Blank A, Schafer SC, Candinas D, Gloor B, Angst E. A retroperitoneal bronchogenic cyst mimicking a pancreatic or adrenal mass. Case Rep Gastroenterol. 2013;7(3):428-32. 
7. Brient C, Muller C, Cassagneau P, Taieb D, Sebag F, Henry JF. A retroperitoneal bronchogenic cyst. J Visc Surg. 2012;149(5):e361-363.

8. Byers JT, Gertz HE, French SW, Wang L. Case report: retroperitoneal bronchogenic cyst as a diagnostic dilemma after colon cancer diagnosis. Exp Mol Pathol. 2018;104(2):158-60.

9. Cao DH, Zheng S, Lv X, Yin R, Liu LR, Yang L, Huang Y, Wei Q. Multilocular bronchogenic cyst of the bilateral adrenal: report of a rare case and review of literature. Int J Clin Exp Pathol. 2014;7(6):3418-22.

10. Mirsadeghi A, Farrokhi F, Fazli-Shahri A, Gholipour B. Retroperitoneal bronchogenic cyst: a case report. Med J Islam Repub Iran. 2014;28:56.

11. Bonhomme A, Broeders A, Oyen RH, Stas M, De Wever I, Baert AL. Cystic lymphangioma of the retroperitoneum. Clin Radiol. 2001;56(2):156-8.

12. Liang MK, Marks JL. Congenital bronchogenic cyst in the gastric mucosa. J Clin Pathol. 2005;58(12):1344.

13. Wang M, He X, Qiu X, Tian C, Li J, Lv M. Retroperitoneal bronchogenic cyst resembling an adrenal tumor with high levels of serum carbohydrate antigen 19-9: a case report. Medicine (Baltimore). 2017;96(31):e7678

14. Choi KK, Sung JY, Kim JS, Kim MJ, Park H, Choi DW, Choi SH, Heo JS. Intra-abdominal bronchogenic cyst: report of five cases. Korean J Hepatobiliary Pancreat Surg. 2012;16(2):75-9.

15. Ishikawa T, Kawabata G, Okada H, Arakawa S, Kamidono S, Fujisawa M. Retroperitoneal bronchogenic cyst managed with retroperitoneoscopic surgery. J Urol. 2003;169(3):1078-9.

16. Buckley JA, Siegelman ES, Birnbaum BA, Rosato EF. Bronchogenic cyst appearing as a retroperitoneal mass. AJR Am J Roentgenol. 1998;171(2):527-8.

17. Yamamoto E, Nakayama H, Ozaki N, Kitamura Y, Funatsuka M, Ueda M, Chikugo K, Hirata A, Kurashina A, Kuratsuka H, et al. Laparoscopically resected foregut cyst adjacent to the right adrenal gland. Diagn Ther Endosc. 1998;5(1):53-6.

18. Itoh H, Shitamura T, Kataoka H, Ide H, Akiyama Y, Hamasuna R, Hasui Y, Osada Y, Koono M. Retroperitoneal bronchogenic cyst: report of a case and literature review. Pathol Int. 1999;49(2):152-5.

19. Sullivan SM, Okada S, Kudo M, Ebihara Y. A retroperitoneal bronchogenic cyst with malignant change. Pathol Int. 1999;49(4):338-41.

20. Yang SW, Linton JA, Ryu SJ, Shin DH, Park CS. Retroperitoneal multilocular bronchogenic cyst adjacent to adrenal gland. Yonsei Med J. 1999:40(5):523-6.

21. Reichelt O, Grieser T, Wunderlich H, Moller A, Schubert J. Bronchogenic cyst. A rare differential diagnosis of retroperitoneal tumors. Urol Int. 2000;64(4):216-9.

22. Haddadin WJ, Reid R, Jindal RM. A retroperitoneal bronchogenic cyst: a rare cause of a mass in the adrenal region. J Clin Pathol. 2001;54(10):801-2.

23. Anderson MI, O'Reilly KJ, Costabile RA. Retroperitoneal bronchogenic cyst mimicking a pheochromocytoma. J Urol. 2001;166(4):1379-80.

24. Martin R, Sanz E, de Vicente E, Ortega P, Labrador E, Paumard A, Gomez-Duran J, Quijano Y, Santamaria L. Differential diagnosis of asymptomatic retroperitoneal cystic lesion: a new case of retroperitoneal bronchogenic cyst. Eur Radiol. 2002;12(4):949-50.

25. Ingu A, Watanabe A, Ichimiya Y, Saito T, Abe T. Retroperitoneal bronchogenic cyst: a case report. Chest. 2002;121(4):1357-9.

26. Andersson R, Lindell G, Cwikiel W, Dawiskiba S. Retroperitoneal bronchogenic cyst as a differential diagnosis of pancreatic mucinous cystic tumor. Dig Surg. 2003;20(1):55-7.

27. Hedayati N, Cai DX, McHenry CR. Subdiaphragmatic bronchogenic cyst masquerading as an "Adrenal incidentaloma." J Gastrointest Surg. 2003;7(6):802-4.

28. Hisatomi E, Miyajima K, Yasumori K, Okamura H, Nonaka M, Watanabe J, Muranaka T, Mori H. Retroperitoneal bronchogenic cyst: a rare case showing the characteristic imaging feature of milk of calcium. Abdom Imaging. 2003;28(5):716-20.

29. Ishizuka O, Misawa K, Nakazawa M, Nishizawa O. A retroperitoneal bronchogenic cyst: laparoscopic treatment. Urol Int. 2004;72(3):269-70.

30. Goh BK, Chan HS, Wong WK. A rare case of "giant" right-sided retroperitoneal bronchogenic cyst. Dig Dis Sci. 2004;49(9):1491-2.

31. Paik SS, Jang KS, Han HX, Oh YH, Lee KG, Choi D. Retroperitoneal bronchogenic cyst mimicking pancreatic pseudocyst in a patient with colorectal cancer. J Gastroenterol Hepatol. 2005;20(5):802-3.
32. Wang SE, Tsai YF, Su CH, Shyr YM, Lee RC, Tsai WC, Li FY, Chen TH, Wu CW, Lui WY. Retroperitoneal bronchogenic cyst mimicking pancreatic cystic lesion. J Chin Med Assoc. 2006;69(11):538-42.

33. Kim EY, Lee WJ, Jang KT. Retroperitoneal bronchogenic cyst mimicking a pancreatic cystic tumour. Clin Radiol. 2007:62(5):491-4.

34. Chu PY, Hwang TI, Teng TH, Lee CC. A retroperitoneal bronchogenic cyst successfully treated by laparoscopic surgery. Ann Saudi Med. 2007;27(3):199-200.

35. Roma A, Varsegi M, Magi-Galluzzi C, Ulbright T, Zhou M. The distinction of bronchogenic cyst from metastatic testicular teratoma: a light microscopic and immunohistochemical study. Am J Clin Pathol. 2008;130(2):265-73

36. Chung JM, Jung MJ, Lee W, Choi S. Retroperitoneal bronchogenic cyst presenting as adrenal tumor in adult successfully treated with retroperitoneal laparoscopic surgery. Urology. 2009;73(2):442. e413-42. e415.

37. Obando J, Merkle E, Bean SM. A retroperitoneal bronchogenic cyst. Clin Gastroenterol Hepatol. 2009;7(8):A21-4.

38. Önol F, Baytekin F, Dikbaş O, Ergönenç T, Tanidir Y. A retroperitoneal bronchogenic cyst mimicking adrenal tumour in an adult: is differential diagnosis truly possible? J Clin Pathol. 2009;62(2):187-9.

39. Inaba K, Sakurai Y, Umeki Y, Kanaya S, Komori Y, Uyama I. Laparoscopic excision of subdiaphragmatic bronchogenic cyst occurring in the retroperitoneum: report of a case. Surg Laparosc Endosc Percutan Tech. 2010;20(6):e199-203.

40. El Youssef R, Fleseriu M, Sheppard BC. Adrenal and pancreatic presentation of subdiaphragmatic retroperitoneal bronchogenic cysts. Arch Surg. 2010;145(3):302-4.

41. Diaz Nieto R, Naranjo Torres A, Gomez Alvarez M, Ruiz Rabelo JF, Perez Manrique MC, Ciria Bru R, Valverde Martinez A, Roldan de la Rua J, Alonso Gomez J, Rufian Pena S. Intraabdominal bronchogenic cyst. J Gastrointest Surg. 2010;14(4):756-8.

42. Petrina A, Boselli C, Cirocchi R, Covarelli P, Eugeni E, Badolato M, Finocchi L, Trastulli S, Noya G. Bronchogenic cyst of the ileal mesentery: a case report and a review of literature. J Med Case Rep. 2010;4:313.

43. Alguraan Z, Agcaoglu O, El-Hayek K, Hamrahian AH, Siperstein A, Berber E. Retroperitoneal masses mimicking adrenal tumors. Endocr Pract. 2012;18(3):335-41.

44. Parray FQ, Sherwani AY, Dangroo SA, Bisati RA, Malik NS. Retroperitoneal bronchogenic cyst mimicking hydatid liver: a case report. Case Rep Surg. 2012;2012:312147.

45. O'Neal PB, Moore FD, Gawande A, Cho NL, King EE, Moalem J, Ruan D. Bronchogenic cyst masquerading as an adrenal tumor: a case of mistaken identity. Endocr Pract. 2012;18(5):e102-105.

46. Cai Y, Guo Z, Cai Q, Dai S, Gao W, Niu Y, Li G, Zhang Y. Bronchogenic cysts in retroperitoneal region. Abdom Imaging. 2013;38(1):211-4.

47. Castro R, Oliveira MI, Fernandes T, Madureira AJ. Retroperitoneal bronchogenic cyst: MRI findings. Case Rep Radiol. 2013;2013:853795.

48. Kluger MD, Tayar C, Belli A, Salceda JA, van Nhieu JT, Luciani A, Cherqui D. A foregut cystic neoplasm with diagnostic and therapeutic similarities to mucinous cystic neoplasms of the pancreas. JOP. 2013;14(4):446-9.

49. Dong B, Zhou H, Zhang J, Wang Y, Fu Y. Diagnosis and treatment of retroperitoneal bronchogenic cysts: a case report. Oncol Lett. 2014;7(6):2157-9

50. Terasaka T, Otsuka F, Ogura-Ochi K, Miyoshi T, Inagaki K, Kobayashi Y, Nasu Y, Makino H. Retroperitoneal bronchogenic cyst: a rare incidentaloma discovered in a juvenile hypertensive patient. Hypertens Res. 2014;37(6):595-7.

51. Bulut G, Bulut M, Bahadır I, Kotan Ç. Bronchogenic cyst mimicking an adrenal mass in the retroperitoneal region: report of a rare case. Indian J Pathol Microbiol. 2015;58(1):96.

52. Herek $D$, Erbis $H$, Kocyigit $A$, Yagci AB. Retroperitoneal bronchogenic cyst originating from diaphragmatic crura. Indian J Surg. 2015;77(Suppl 3):1397-8.

53. Jiang $X$, Zeng H, Gong J, Huang R. Unusual uptake of radioiodine in a retroperitoneal bronchogenic cyst in a patient with thyroid carcinoma. Clin Nucl Med. 2015;40(5):435-6.

54. Robertson FP, Tsironis D, Davidson BR. A diaphragmatic retroperitoneal cyst. Ann R Coll Surg Engl. 2015;97(5):e77-78.

55. Tong HX, Liu WS, Jiang Y, Liu JU, Zhou JJ, Zhang Y, Lu WQ. Giant retroperitoneal bronchogenic cyst mimicking a cystic teratoma: a case report. Oncol Lett. 2015;9(6):2701-5. 
56. Trehan M, Singla S, Singh J, Garg N, Mahajan A. A rare case of intraabdominal bronchogenic cyst-a case report. J Clin Diagn Res. 2015;9(11):PD03-04.

57. Yoon YR, Choi J, Lee SM, Kim YJ, Cho HD, Lee JW, Jeon YS. Retroperitoneal bronchogenic cyst presenting paraadrenal tumor incidentally detected by (18)F-FDG PET/CT. Nucl Med Mol Imaging. 2015;49(1):69-72.

58. Pasquer A, Djeudji F, Hervieu V, Rabeyrin M, Barth X. A rare retrorectal presentation of a bronchogenic cyst: a case report. Int I Surg Case Rep. 2016;24:112-4.

\section{Publisher's Note}

Springer Nature remains neutral with regard to jurisdictional claims in published maps and institutional affiliations.
Ready to submit your research? Choose BMC and benefit from:

- fast, convenient online submission

- thorough peer review by experienced researchers in your field

- rapid publication on acceptance

- support for research data, including large and complex data types

- gold Open Access which fosters wider collaboration and increased citations

- maximum visibility for your research: over 100M website views per year

At BMC, research is always in progress.

Learn more biomedcentral.com/submissions 